\title{
Utilisation of plasma centrifuges for life support systems on Mars
}

\author{
J. Gruenwald ${ }^{1}$ \\ ${ }^{1}$ Gruenwald Laboratories, Taxberg 50, 5660 Taxenbach, Austria
}

(Received: 03. December 2018, Accepted: 11. December 2018, Published online: 12. December 2018)

In this paper the possibility of utilising a plasma centrifuge for oxygen generation in outer space is discussed. It is proposed that a plasma centrifuge can not only create oxygen for human consumption very efficiently but is also able to produce useful by-products. Special emphasis is given to life support systems working in the atmosphere of Mars, where oxygen and carbon raw materials can be obtained directly from the atmosphere. The system under consideration in this work is a plasma centrifuge with axial circulation that contains a fully ionised plasma. Under these conditions the carbon dioxide from the Mars atmosphere will be entirely dissociated. Thus, the atomic oxygen and carbon can easily be separated. (c) G-Labs 2018

(DOI 10.31281/jtsp.v1i1.4)

jgruenwald@gmx.at

\section{Introduction}

Using centrifugal force and plasma to dissociate and separate molecules from the Mars atmosphere or from $\mathrm{CO}_{2}$ that is exhaled by humans, has been proposed by different authors over the last view years [1-5]. While these authors suggested classical gas centrifuges with a mechanical rotor, the work presented here will focus on the use of plasma centrifuges (PC). The physical principal of isotope separation with a plasma was experimentally demonstrated by Bonnevier in the 1970'ies [6]. Since this pioneering work PCs have been examined for partially [7,8] and fully ionised [9-11] gases. The most recent advancement in the field of PCs has been the introduction of an additional axial flow. It has been shown that a PC with such an additional circulation has a much higher separation power than conventional ones [12-14] or PCs with only radial separation. These papers by previous authors show that there are some limitations for conventional gas centrifuges that are no issue by PCs. It is impossible, for example, to separate isotopes with a low vapour pressure in classical centrifuges. Hence, the PC separation of calcium isotopes was put forward in Ref. [14]. Another issue of purely mechanical centrifuges is the high cost connected with the separation of isotopes of light noble gases such as helium or neon.
PCs have yet another additional feature that has to the knowledge of the author not been discussed before. They are able to turn at least a part of the separated gas particles into useful byproducts via chemical vapour deposition (CVD) or plasma enhanced chemical vapour deposition (PECVD). This is particularly the case for carbon containing gases such as $\mathrm{CO}$ and $\mathrm{CO}_{2}$, which can be used to deposit numerous carbon phases such as graphene, graphite or diamond. These deposition processes are used already frequently here on earth and it was proposed in Refs. [2, 3] to use plasma and centrifugal forces to obtain oxygen and carbon based raw materials from the Mars atmosphere. The objective of this paper is to investigate the possibility of applying a PC instead of conventional centrifuges in this previously suggest life support technology. It shall also be noted that such a hybrid system of PC and deposition reactor can, in principle, not only be used for manned Mars missions but also for life support on long term space missions and here on Earth for coating/deposition technologies and for $\mathrm{CO}_{2}$ sequestration from the Earth atmosphere. Another interesting field of application might be the usage of such a system for chemical treatment of exhaled air in submarines, where the need for compact air recycling systems is very high. 


\section{Physical and Technical Parameters}

The following Fig. 1 depicts an exemplary schematic drawing of a PC with axial flow separation. It has to be emphasised that in this paper the means of plasma creation (e.g. rf, microwave, etc.) are not considered. This paper is supposed to be only a starting point for the construction of plasma technology base life support systems and is focused on the calculation of the most important technical and physical parameters. The comparison between different mechanisms of plasma creation and the exact way of producing axial flows is left for future work. However, rf waves or high-frequency rotating magnetic fields for inducing axial flows have been proposed in a previous work $[12,13]$. For this work the creation of axial flows by a travelling magnetic field as described in [13] is considered.

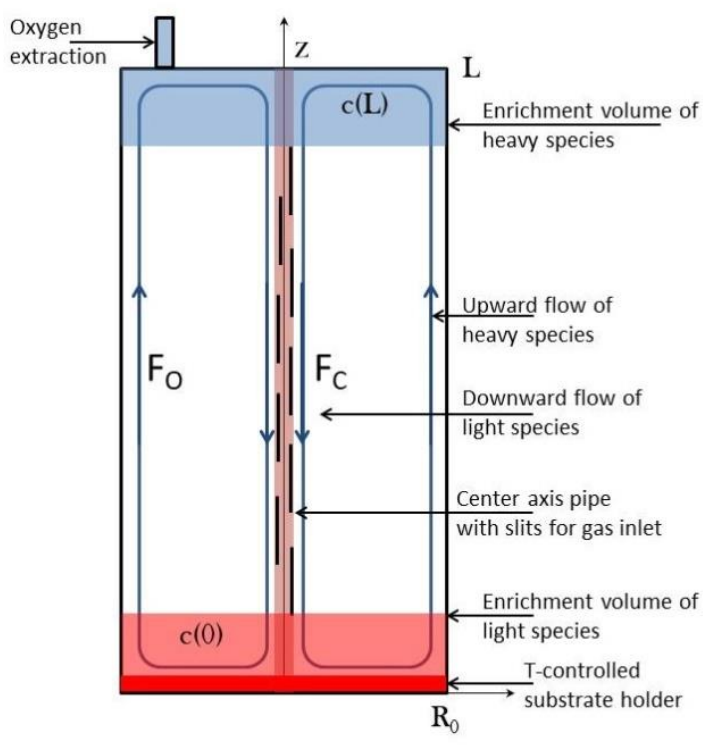

Figure 1: This is an example for a single column figure.

For the following calculations we assume a fully ionised plasma in a cylindrical centrifuge chamber. This has the advantage that all molecules (i.e. $\mathrm{CO}_{2}$ from the Mars atmosphere) are fully dissociated into carbon and oxygen atoms and the content of the PC is a binary mixture. Furthermore, it is assumed that the axial separation flows are fully established and have reached a steady state condition. The cylindrical $P C$ has an inner radius of $R_{0}$ and a height denoted with $L$. The feed gas will be introduced via a pipe with slits, which is situated at the central axis of the chamber. As shown by Borisevich and Potanin [13], the axial flows will transport the heavier species to the top of the vessel while the lighter species will accumulate at the bottom. Hence, the oxygen and carbon plasma flows are denoted with $F_{O}$ and $F_{C}$ respectively. The concentration of the separated particles is written as $c(0)$ for the lower part of the machine and $c(L)$ at the ceiling. As oxygen has the higher molar mass $\mathrm{M}(\sim 16 \mathrm{~g} / \mathrm{mol})$ than carbon $(\sim 12 \mathrm{~g} / \mathrm{mol})$, it can easily be extracted from the top of the PC vessel. Carbon, on the other hand, can be deposited on a temperature controlled substrate on the bottom of the chamber. The temperature control is highly important since the substrate temperature determines the form of carbon phase that is deposited. This enables the on-side production of a large variety of carbon materials, which will be extremely beneficial in future long term space missions or even the colonisation of Mars. Since the latter is sincerely considered by a growing number of companies, NGOs and governments the PC might play an important role on such endeavours. The basic technical and physical parameters of such a system will be presented in this section.

The first entity to look at is the plasma rotation frequency $\Omega$. If there is no diamagnetic drift, $\Omega$ is given by [12]:

$$
\Omega=\frac{\Omega_{i}}{2}\left(\sqrt{1+4 \frac{\Omega_{E}}{\Omega_{i}}}-1\right)
$$

With $\Omega_{\mathrm{i}}$ and $\Omega_{\mathrm{e}}$ are the ion cyclotron and the ExB rotation frequency, respectively. They are defined via:

$$
\Omega_{i}=\frac{q B}{m}
$$

and

$$
\Omega_{E}=-\frac{E}{r B}
$$

The radial position is to be taken at the vicinity of the chamber wall. Hence, $r=R_{0}=0.25 \mathrm{~m}$, according to [3]. Inserting the following values for the electrical field $E=5000 \mathrm{~V} / \mathrm{m}$, the mass of the carbon ion $m_{c}=1.99 \times 10^{-26} \mathrm{~kg}$, the mass of the oxygen ion $m_{O}=2.66 \times 10^{-26} \mathrm{~kg}$ and the magnetic field B into Eqs. (1)-(3), one obtains the results shown in the following Fig. 2 for singly charged (i.e. $q=1$ ) ions: 


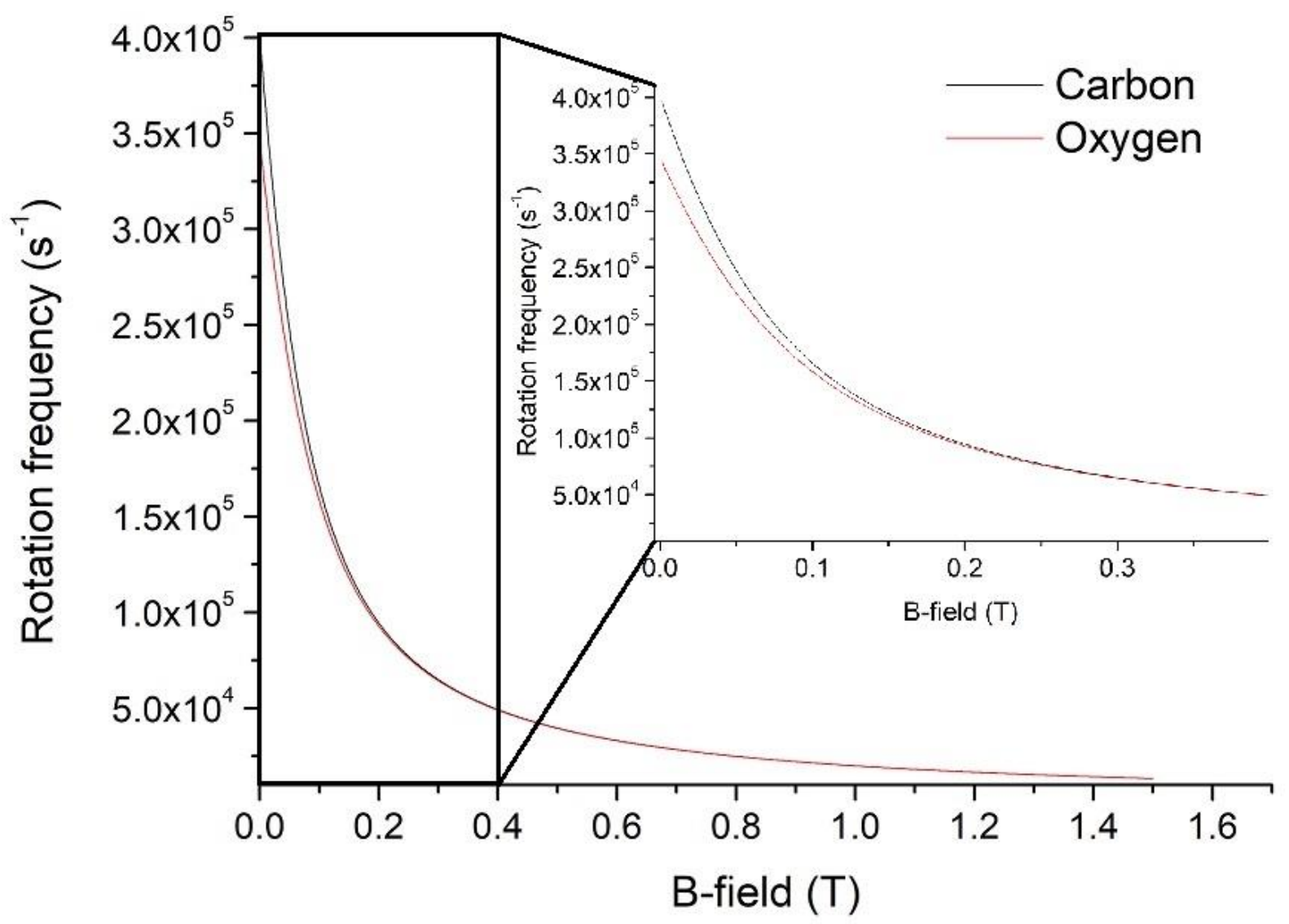

Figure 2: Plasma rotation frequency of carbon (black) and oxygen (red) for a chamber radius of $0.25 \mathrm{~m}$ as a function of the applied B-field. Inlay: Magnified view on the rotation frequency in magnetic field regions up to $0.4 \mathrm{~T}$.

Fig. 2 displays the calculated plasma rotation frequencies for oxygen and carbon ions in a range between 0 and $1.5 \mathrm{~T}$ that covers the typical maximum field strength of rare earth permanent magnets (up to $1.4 \mathrm{~T}$ for $\mathrm{Nd}_{2} \mathrm{Fe}_{14} \mathrm{~B}$ ). It is evident that for high magnetic field strength the curves converge because of the dominance of the ion cyclotron frequency, which is independent of the mass of the ions. However, it has to be noted that the technically most relevant range of magnetic field strength is depicted in the inlay of Fig. 2 between 0 and $0.4 \mathrm{~T}$, which yields a plasma rotation frequency in the order of $10^{5} \mathrm{~Hz}$. This high rotation frequency can be achieved by suitable radiofrequency waves. As discussed by Fetterman and Fisch [12], the upper power limit for the excitation of such a wave is connected to the gas flow $\mathrm{F}$ into the PC via:

$$
P=\frac{F \Omega^{2} R_{0}^{2}}{4}
$$

It was pointed out in Ref. [2] that the oxygen consumption of an average human is about $4 \mathrm{~m}^{3}$ per day, which corresponds to a flow of 3000 $\mathrm{sccm}$ or $0.0315 \mathrm{~g} / \mathrm{s}$. Thus, a life support system for Mars missions based on a PC would require $1.7 \mathrm{~kW}$ input power to induce a wave driven plasma rotation in an exemplary magnetic field of $0.33 \mathrm{~T}$. This has to be added to the energy requirements for the plasma generation and is considerably more than the energy consumption of a classical centrifuge (about $4.5 \mathrm{~kW}$ [3]). On a first glance this would seem disadvantageous but one has also to take into account the maximum separation power of a PC compared to a regular mechanical one. This can be estimated by the following equation given by Fetterman [12]:

$$
\frac{\partial U_{\text {plasma }}}{\partial U_{\text {gas }}} \approx \frac{\sqrt{T_{i}}}{\frac{\ln \left(N_{D}\right)}{10}} \cdot \frac{\kappa^{2}}{1+\kappa^{2}}\left(\frac{\Omega R_{0}}{10 v_{a}}\right)
$$

with

$$
\kappa=\frac{1}{\Omega_{i} \tau_{i}}
$$


Here $T_{i}$ is the ion temperature in $e V, \ln \left(N_{D}\right)$ is the natural logarithm of the Debye number $N_{D}, v_{a}$ $=\omega_{\text {centrifuge }} \times R_{0}$, is the peripheral speed of the gas centrifuge and $\tau_{i}$ is the ion-ion collision time. The ion-ion collision time and the Debye number are calculated according to [15]:

$$
\begin{aligned}
& \ln \left(N_{D}\right)=18.4-1.15 \log _{10}\left(n_{e}\right)+ \\
& 2.3 \log _{10}\left(T_{e}\right) \\
& \tau_{i}=3.44 \cdot 10^{11} T_{e}^{3 / 2} \frac{1}{\ln \left(N_{D}\right)}\left(\frac{m_{i}}{m_{e}}\right)^{1 / 2}
\end{aligned}
$$

where $n_{e}$ has to be taken in $\mathrm{m}^{-3}$ while $T_{e}$ is in $e V$. The electron density and temperature as well as the ion temperature and mass are determined as follows:

The Mars atmosphere has an average pressure of $636 \mathrm{~Pa}$ at $240 \mathrm{~K}$ [16]. This corresponds to a neutral gas density of $1.9 \times 10^{23} \mathrm{~m}^{-3}$. As a fully dissociated and ionised gas is assumed for our calculations, there will be $1.9 \times 10^{23} \mathrm{C}$ atoms per $\mathrm{m}^{3}, 3.8 \times 10^{23} \mathrm{O}$ atoms per $\mathrm{m}^{3}$ and $5.7 \times 10^{23}$ electrons per $\mathrm{m}^{3}$. Furthermore, Ellis et al. measured electron temperatures of $\mathrm{T}_{\mathrm{e}} \sim 1-2.5 \mathrm{eV}$ and ion temperatures of $\mathrm{T}_{\mathrm{i}} \sim 30 \mathrm{eV}$ in a plasma with $100 \%$ ionisation rate [8]. This yields a $\ln \left(\mathrm{N}_{\mathrm{D}}\right)$ of 5.7. Thus, the ion-ion collision time is $3.1 \times 10^{-7}$ s. Accordingly, $\mathrm{k}=1.4$ for a magnetic field of 0.33 $\mathrm{T}$, where $\Omega_{\mathrm{i}} \approx 2.3 \times 10^{6} / \mathrm{s}$. In accordance with Eq. (5) the ratio of the maximal separation powers is 22. This indicates that a single PC can replace 22 conventional centrifuges in terms of radial separation power. It has to be taken into account that due to the heating of the ions the particle density will drop about 2-3 orders of magnitude after reaching a steady state operation with the PC, which will have to be taken into account in the next considerations.

For the following calculations the magnetic Reynolds number $\mathrm{Re}_{\mathrm{m}}$ and the dimensionless parameter $\chi$ will be needed. The former is defined via:

$$
R e_{m}=\mu_{0} \cdot \sigma \cdot \omega_{0} \cdot R_{0}
$$

where $\mu_{0}$ is the vacuum permeability, $\sigma$ is the plasma conductivity and $\omega_{0}$ is the magnetic field rotation frequency. $X$ is given by [14]:

$$
\chi=\operatorname{ber}_{0}^{2}\left(\sqrt{R e_{m}}\right)+b e i_{0}^{2}\left(\sqrt{R e_{m}}\right)
$$

Here ber $_{0}$ and bei $_{0}$ are the Kelvin functions. The plasma conductivity can be calculated with the electron density and the electron-electron collision frequency $v_{\text {ee }}$ via:

$$
\sigma=\frac{n_{e} \cdot e^{2}}{m_{e} \cdot v_{e e}}
$$

The electron-electron collision frequency is obtained with the equation [19]:

$$
v_{e e}=5 \cdot 10^{-6} \frac{n_{e} \ln \left(N_{D}\right)}{T_{e}^{3 / 2}}
$$

With an electron-electron collision time of $3.6 \mathrm{x}$ $10^{10} / \mathrm{s}$ for the electron density of $5.7 \times 10^{17}$ electrons per $\mathrm{cm}^{3}$, one obtains a plasma conductivity of $3.9 \times 10^{3} \mathrm{~S} / \mathrm{m}$. The magnetic Reynolds number is calculated as a function of the chamber radius for different rotation frequencies of the B-field. The results are shown in the following Fig. 3:

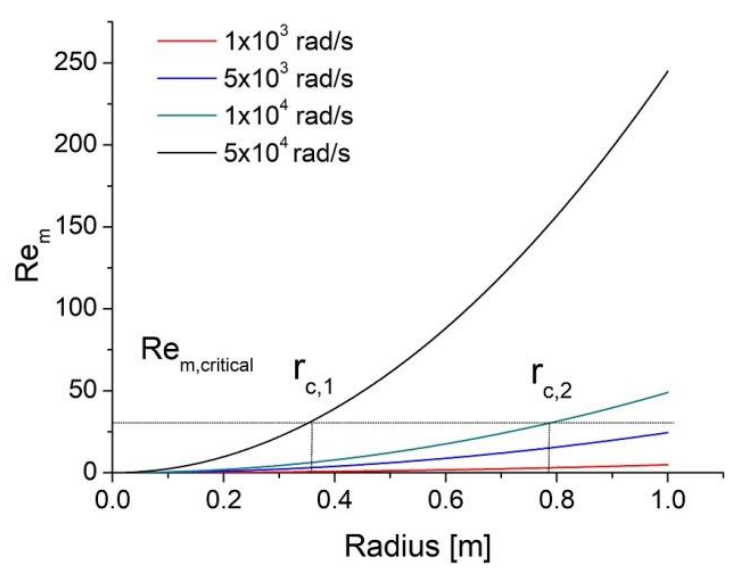

Figure 3: magnetic Reynolds number as a function of centrifuge radius for different rotation speeds of the magnetic field.

For $\mathrm{Re}_{\mathrm{m}}<1$ the eddy current braking is similar to the one of an induction motor but if $\mathrm{Re}_{\mathrm{m}}>30$ much more energy is dissipated in the plasma and the eddy braking torque decreases much slower for higher rotation speed than in an induction motor [17]. Thus, the critical radii for a PC chamber are depicted in Fig. 3 for high rotation frequencies of the magnetic field. A rotation frequency of $10^{4} \mathrm{rad} / \mathrm{s}$ corresponds to a critical radius of $78.6 \mathrm{~cm}$, while a $5 \times 10^{4} \mathrm{rad} / \mathrm{s}$ correspond to a critical radius of $35.5 \mathrm{~cm}$. Therefore, it is evident that the higher $\omega_{0}$, the smaller the radius of the chamber. Hence, for an assumed $\mathrm{Re}_{\mathrm{m}} \sim 0.5 \chi=1.121$.

In accordance with Refs. $[13,14]$ the radial and axial enrichment factors $\left(\varepsilon_{\mathrm{r}}\right.$ and $\left.\varepsilon_{\mathrm{z}}\right)$ are calculated as follows: 


$$
\varepsilon_{r}=\frac{\Delta M B_{0}^{4} R_{0}^{2} R e_{m}^{2}}{1024 \eta^{2} \mu_{0}^{2} \Re T \chi^{2}}
$$

Where $\Delta M$ is the difference in the molar masses, $\eta$ is the dynamic viscosity of the working gas and $\mathfrak{R}$ is the ideal gas constant. The dynamic viscosity can be calculated from the product of kinematic viscosity $\xi$ and the particle density. $\xi$ is given as a function of the thermal velocity $v_{t}$ for a fully ionised plasma via [18]:

$$
\xi=\frac{v_{t}^{2}}{v_{i i}}
$$

Here the average thermal velocity is about $2 \times 10^{4}$ $\mathrm{m} / \mathrm{s}$ for ions with $T_{i} \sim 30 \mathrm{eV}$, which gives a dynamic viscosity of around $1.7 \times 10^{-2} \mathrm{~kg} / \mathrm{m} \cdot \mathrm{s}$. This yields a radial enrichment factor of 0.43 . On the other hand, the axial enrichment factor for a $P C$ with the length $L$ is defined by:

$$
\varepsilon_{z} \approx 12 \varepsilon_{r} \frac{V N_{1}}{\left(2 V^{2} N_{2}+1\right)} \frac{L}{R_{0}}
$$

with the contributions

$$
\begin{gathered}
N_{1}=\int_{0}^{1}\left[y\left(1-y^{2}\right)\right]^{2} \psi_{0}^{2} d y \\
N_{2}=\int_{0}^{1} \psi_{0}^{2} \frac{1}{y} d y \\
V=\frac{\rho^{*} V_{z 0} R_{0}}{2\langle\rho D\rangle} \frac{G^{2}}{(1+(G-1) \exp (G))} \\
\psi_{0}=\exp (G y)\left[\lambda\left(y^{2}-1\right)+1-y^{4}\right]
\end{gathered}
$$

where $\mathrm{G}$ is the compressibility of the working gas, $\rho^{*}$ is the gas mass density at elevated temperature $\left(1.4 \times 10^{-4} \mathrm{~kg} / \mathrm{m}^{3}\right.$, assumed to be constant over the whole volume in steady state), $D$ is the self-diffusion value of the working gas $\left(1.56 \times 10^{-3} \mathrm{~m}^{2} / \mathrm{s}[3]\right),<\ldots>$ denotes the mean value, $y=r / R_{0}$ is a normalised radial position, $\psi_{0}$ is a stream function and $\lambda$ is a dimensionless parameter. $\mathrm{V}_{\mathrm{z} 0}$ is defined by:

$$
V_{z 0}=\frac{\sigma B_{0}^{2} R_{0}^{4} k_{z} \omega_{1} R e_{m}}{128 \eta\left(f_{1}^{2}+f_{2}^{2}\right)}
$$

where $\mathrm{f} 1$ and $\mathrm{f} 2$ are dimensionless parameters, as defined in [13] and are of the order 1. $\omega_{1}$ is the angular frequency of the of the travelling magnetic field, which is considered to be of the order of $10^{4} \mathrm{rad} / \mathrm{s}$ and $\mathrm{k}_{\mathrm{z}}=\omega_{1} / \mathrm{v}_{\mathrm{ph}}=0.2 / \mathrm{m}$. The phase velocity of the wave is considered to be about $5 \times 10^{4} \mathrm{~m} / \mathrm{s}$ for our example. This yields with Eq. (20) $V_{z 0}=589.1 \mathrm{~m} / \mathrm{s}$. Since, we consider $\mathrm{a}$ fully ionised plasma with strong Coulomb interactions and spatially constant density the compressibility will be $G=0$ and $\langle\rho D\rangle=\rho D$. If $G$ vanishes, $\lambda=4 / 3$ [13] and the second fraction on the r.h.s. of Eq. (18) converges to 2.

Hence, the stream function from Eq. (19) becomes:

$$
\psi_{0}=\left[\frac{4}{3}\left(y^{2}-1\right)+1-y^{4}\right]
$$

The results for the stream function in dependence of the normalized radial position are depicted in the following Fig. 4:

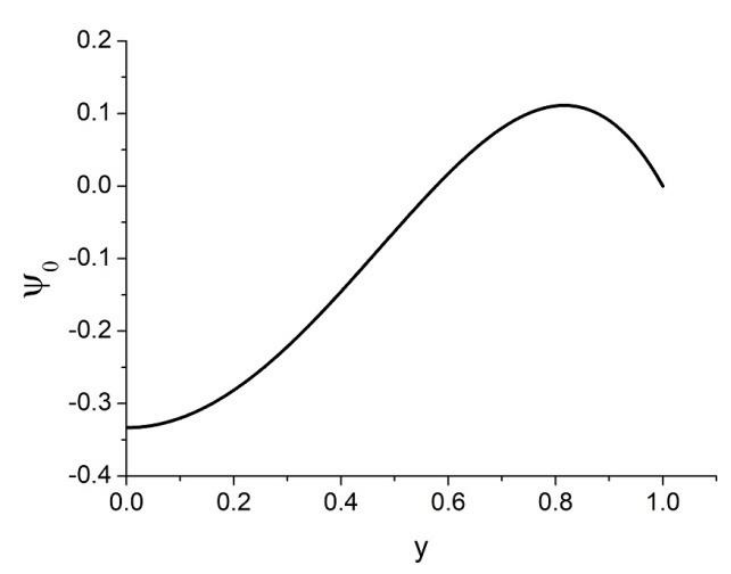

Figure 4: Simulated stream function in dependence on the normalised radial position within the PC vessel.

It is evident that the maximum of the stream function occurs in the close vicinity of the edge of the centrifuge vessel. Using the relations $\rho^{*}$ $0.01 \rho$ and $\langle\rho D>\sim<\eta>$ Eq. (18) then gets simplified to:

$$
V=\frac{\rho V_{z 0} R_{0}}{100\langle\eta\rangle}=1.21
$$

and $N_{1}=1.26 \times 10^{-3}, N_{2}=0.62$. With these data the longitudinal enrichment factor becomes for a PC with a length of $1 \mathrm{~m} \varepsilon_{z} \sim 1.1 \times 10^{-2}$. This below the radial enrichment factor in our example but the value of $\varepsilon_{\mathrm{z}}$ can be improved considerably by increasing the ratio $L / R_{0}$. This enables not only the efficient production of oxygen but also the creation of high-purity carbon phases as part of the process.

Under optimal operation conditions, the logarithm of the separation factor $\ln (a)$ can be obtained via [14]:

$$
\ln (\alpha)=\exp \left(\varepsilon_{Z} L_{1} N \frac{1-\exp \left(-L_{1} h / 4 \pi\right)}{L_{1} h / 4 \pi}\right)
$$


In Eq. (23) $N$ denotes the ratio $N_{1} / N_{2}$, which is defined by in [14]. In our case $N=2.3 \times 10^{-3} . L_{1}=$ $L_{p} / R_{0}$ where $L_{p}$ is the length of the enriching part of the whole reactor length. $h=P /\left(<\rho D>R_{0}\right)$ where $P$ denotes the Product flow rate, i.e. the breathable oxygen mass flow created by the life support system. An exemplary calculation of the logarithm of the separation factor is shown in the following Fig. 5 for $L_{1}=5$.

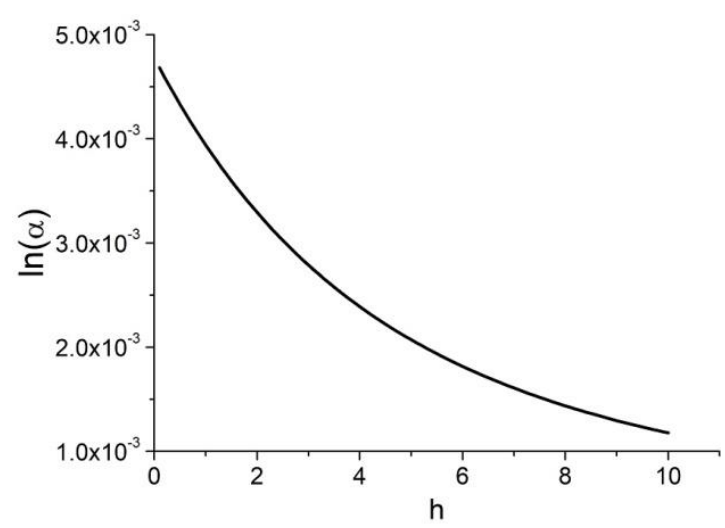

Figure 5: Logarithm of the separation factor as a function of the parameter $h$ with $L_{1}=5$.

Despite the fact that $\ln (a)$ is rather small in this example, it has to be kept in mind that the calculations in this paper have been done for a life support system with a rather large radius and short height as it was proposed in Refs. $[2,3]$ in order to get a direct comparison. Thus, it is emphasised at this point that there is a lot of room for optimisation by choosing longer, thinner PC chambers, which will enhance the enrichment and separation coefficients considerably.

\section{Conclusion}

The usage of PCs for oxygen and carbon raw material creation with a strong focus on Mars life support systems has been presented in this work. It was demonstrated that this type of centrifuge has much higher separation and enrichment factors as classical, mechanical centrifuges. It is, thus, very reasonable to argue that this kind of life support can play a crucial role in future long term space missions but may also be used here on earth, for example, in submarines. The combination of plasma enhanced $\mathrm{CO}_{2}$ dissociation, separation and deposition will be extremely beneficial in space, especially on manned Mars habitats, where a high degree of on-side production technologies will be needed. A direct comparison between a classical centrifuge and a PC shows that the main advantage of the latter so far is the high separation power. The enrichment and separation factors, on the other hand, can be extremely improved by adapting the geometry of the PC vessel. Especially the aspect ratio $L / R_{0}$ has to be substantially increased to obtain an even better separation in a plasma centrifuge. This is a striking difference to an ordinary, mechanical centrifuge, where it has been shown in previous work by the author that a bulky ellipsoidal gas centrifuge with a height of 1 $\mathrm{m}$ and a diameter of $0.5 \mathrm{~m}$ can deliver enough breathable oxygen for about 10 average humans. On the contrary, a PC must be designed a long, rather thin pipe in order to obtain an optimised output, but if done properly one PC may be able to replace some dozens of mechanical centrifuges, while producing quite substantial amounts of carbon based raw materials directly from the Mars atmosphere. It shall also be pointed out that such a plasma based life support system can be easily combined with other life support systems, especially with those that use microorganisms and create hydrogen as a byproduct. A combination of such reactors enables an even broader range of raw materials that can be produced. Further considerations of technical details and engineering problems are left for future work.

\section{References}

[1] J. Gruenwald, Human outposts on Mars: engineering and scientific lessons learned from history, CEAS Space J. 6, 73. 2014 https://doi.org/10.1007/s12567-014-0059-8

[2] J. Gruenwald, A proposal for Plasma Technology based Hybrid Life Support System for future Mars Habitats, J. Space Tech., 4, 1. 2014

[3] J. Gruenwald, A hybrid plasma technology life support system for the generation of oxygen on Mars: Considerations on materials and geometry, Acta Astronautica, 123, 188-191. 2016. https://doi.org/10.1016/j.actaastro.2016.03.0 $\underline{21}$

[4] V. Guerra, Vasco, et al. The case for in situ resource utilisation for oxygen production on Mars by non-equilibrium plasmas. Plasma Sources Sci. Technol. 26, 11. 2017 https://doi.org/10.1088/1361-6595/aa8dcc 
[5] J. Gruenwald Comment on 'The case for in situ resource utilization for oxygen production on Mars by non-equilibrium 028001. 2017 https://doi.org/10.1088/1361-6595/aaa873

[6] B. Bonnevier, "Experimental evidence of element and isotope separation in a rotating plasma, Plasma Phys. 13, 763. 1971 https://doi.org/10.1088/0032-1028/13/9/007

[7] B. Lehnert, The partially ionized plasma centrifuge. Phys. Scr., 7, 3, 102. 1973 https://doi.org/10.1088/0031-8949/7/3/002

[8] R. F. Ellis et al., Steady supersonically rotating plasmas in the Maryland centrifugal experiment. Phys. Plasmas, 12, 55704. 2005 https://doi.org/10.1063/1.1896954

[9] M. Krishnan, M. Geva, and J. L. Hirshfield. Plasma centrifuge. Phys. Rev. Let. 46.1, 36. 1981

https://doi.org/10.1103/PhysRevLett.46.36

[10] A. J. Fetterman, and N. J. Fisch. Wave-driven countercurrent plasma centrifuge. Plasma Sources Sci. and Technol. 18.4, 045003. 2009 https://doi.org/10.1088/0963$\underline{0252 / 18 / 4 / 045003}$

[11] A. A. Ivanov, and N. N. Timchenko. Counterstreaming mass separation of ions in a fully ionized rotating plasma. Sov. J. Plasma Phys 16, 863-6. 1990

[12] A. J. Fetterman and N. J. Fisch, Wave-driven countercurrent plasma centrifuge. Plasma Sources Sci. Technol. 18, 045003. 2009 https://doi.org/10.1088/0963$\underline{0252 / 18 / 4 / 045003}$

[13] V. D. Borisevich and E. P. Potanin, The concept of a plasma centrifuge with a high frequency rotating magnetic field and axial circulation. Phys. Scr. 92, 075601. 2017 https://doi.org/10.1088/1402-4896/aa71d2

[14] V. D. Borisevich, E. P. Potanin and J. Whichello, Plasma centrifuge with axial circulation for calcium isotope separation. Phys. Plasmas 25, 113503. 2018 https://doi.org/10.1063/1.5031703

[15] P. Helander, and D. J. Sigmar, Collisional transport in magnetized plasmas, (Vol. 4). Cambridge University Press. 2005
[16] N. Barlow, Mars: An Introduction to its Interior, Surface and Atmosphere, Cambridge Planetary Science. 2018

[17] M. D. Ripper and V. G. Endean, Eddy current Braking Torque Measurements on a Thick Copper Disc, Proc. IEEE, 122, 3, 1975. https://doi.org/10.1049/piee.1975.0080

[18] A. A. Schekochihin et al., Plasma Instabilities and Magnetic Field Growth in Clusters of Galaxies, The Astrophysical Journal, 629:139-142, 2005 https://doi.org/10.1086/431202

[19] F. F. Chen, Introduction into Plasma Physics and controlled Fusion, $2^{\text {nd }}$ edition, springer, Vol. 1. 2006

\section{(9)}

Open Access. This article is licensed under a Creative Commons Attribution 4.0 International License, which permits use, sharing, adaptation, distribution and reproduction in any medium or format, as long as you give appropriate credit to the original author(s) and the source, provide a link to the Creative Commons license, and indicate if changes were made. The images or other third party material in this article are included in the article s Creative Commons license, unless indicated otherwise in a credit line to the material. If material is not included in the article's Creative Commons license and your intended use is not permitted by statutory regulation or exceeds the permitted use, you will need to obtain permission directly from the copyright holder. To view a copy of this license, visit: http://creativecommons.org/licenses/by/4.0/. 